In all, Alenius et al. recruited 102 healthy controls, 306 patients with psoriasis (160 with arthritis and 146 without), and 101 patients with early RA. As expected, the prevalence of anti-CCP antibodies was highest in patients with early RA (74\%). Among patients with psoriasis, those with arthritis had a higher prevalence of anti-CCP antibodies than those without $(7 \%$ versus $0.7 \%)$. The prevalence of anti-CCP antibodies in controls was $2 \%$.

Notably, at follow-up 4 years later, 8 of the 11 patients with psoriatic arthritis who were anti-CCP-positive fulfilled the American College of Rheumatology criteria for RA: they were RF-positive, and had morning stiffness, polyarthritis, and arthritis in the hands. Five of these eight patients also had clinical signs associated with psoriatic arthritis, however, despite fulfilling the criteria for RA. It is possible these patients had both conditions, say the authors.

Caroline Barranco

Original article Alenius GM et al. (2006) Antibodies against cyclic citrullinated peptide (CCP) in psoriatic patients with or without joint inflammation. Ann Rheum Dis 65: 398-400

\section{Which patients could benefit from early biologic therapy for rheumatoid arthritis?}

Patients with rheumatoid arthritis $(\mathrm{RA})$ experience progressive joint destruction that can be retarded by disease-modifying antirheumatic drugs. Not all patients respond to conventional agents, however; some require costly biologic therapies. Smolen et al. evaluated data from 1,004 patients with active, early RA from the ASPIRE trial cohort, with the aim of identifying markers associated with treatment response (defined as retardation of radiographically visible progression of joint damage). Patients were randomly assigned to receive methotrexate only, or methotrexate plus infliximab, according to the ASPIRE protocol.

They found that methotrexate monotherapy was highly effective at retarding disease progression in patients with normal levels of acute-phase inflammatory markers. Patients receiving methotrexate monotherapy who had high baseline levels of joint damage and disease activity (assessed by swollen-joint count and acute-phase inflammatory markers) were, however, more likely to show radiographically visible progression of joint damage-these patients might be good candidates for early introduction of infliximab, say the authors. By contrast, treatment with methotrexate plus infliximab inhibited progression of joint damage regardless of disease activity or baseline joint damage.

Physicians normally assess response to methotrexate monotherapy after 3-6 months of treatment. Smolen et al. found that high disease activity at week 14 predicted progressive joint damage after 1 year, and suggest that such patients should not continue on methotrexate monotherapy. The authors note, however, that the ASPIRE trial was not specifically designed to identify markers associated with disease progression.

Caroline Barranco

Original article Smolen JS et al. (2006) Predictors of joint damage in patients with early rheumatoid arthritis treated with high-dose methotrexate with or without concomitant infliximab: results from the ASPIRE trial. Arthritis Rheum 54: 702-710

\section{Early, high BMI linked with increased risk of total-hip arthroplasty in patients with OA}

Prior studies have shown that, in patients with primary osteoarthritis $(\mathrm{OA})$, the risk of requiring total-hip arthroplasty rises with increasing age and BMI. Flugsrud and colleagues have shown that, for patients with primary $\mathrm{OA}$, early-onset obesity is associated with greater increases in relative risk of later total-hip arthroplasty than late-onset obesity. Obese individuals, however, maintained an elevated relative risk of total-hip arthroplasty as they aged.

The study compared height and BMI data of 1,152,006 people aged 18-67 years (obtained from a nationwide tuberculosis screening program) with similar data from records of 28,425 total-hip arthroplasties for primary OA, obtained from the Norwegian Arthroplasty Register. Women had more than twice the relative risk of later total-hip arthroplasty compared with men, after adjusting for confounding factors, including age and BMI. Men and women who were obese (BMI $\geq 32 \mathrm{~kg} / \mathrm{m}^{2}$ ) by the age of 25 had a substantially increased relative risk of total-hip arthroplasty, compared with individuals who were obese 